\title{
MANIFESTACIONES NEUROLÓGICAS DEL COVID-19
}

COVID-19 NEUROLOGIC MANIFESTATION

MANIFESTAÇÕES NEUROLÓGICAS DE COVID-19

Sebastián Joaquín Ocampo-Rojas ${ }^{1}$

1 Estudiante de Medicina de 6to año. Universidad Nacional de Asunción. Paraguay.

Dirección de e-mail: sebas13ocampo@gmail.com

ORCID: https://orcid.org/0000-0003-4183-4960

\section{Estimado Director:}

Leyendo su artículo "La pandemia por el nuevo coronavirus covid-19", me gustaría profundizar los aportes de la sintomatología de esta enfermedad. Lo que ha comenzado como una patología manifestada por un síndrome respiratorio como neumonías atípicas, actualmente está asociada a varias manifestaciones no respiratorias ${ }^{(1)}$.

De las primeras manifestaciones neurológicas reportadas está el síndrome de Guillain-Barré en una viajera proveniente de Wuhan ${ }^{(2)}$. La literatura es rica en cuanto a cefaleas, convulsiones e ictus en los pacientes graves, si bien la primera y última de estas pueden deberse a complicaciones sistémicas de este novel coronavirus, se ha reportado una mayor incidencia de eventos en pacientes con antecedentes de manifestaciones neurológicas ${ }^{(3,4)}$.

Una de las más recientes manifestaciones reportadas, son las enfermedades neuromusculares. Guidon et al. critican la falta de rigurosidad en los reportes sobre manifestaciones neuromusculares ${ }^{(4)}$.

Si algo es seguro, una manifestación de esta pandemia es la gran cantidad de información disponible en el ámbito científico, muchas veces de estas sin la misma rigurosidad metodológica anterior a la pandemia, acentuando la responsabilidad del lector de analizar críticamente toda la información.

Palabras clave: infecciones por coronavirus; enfermedades del sistema nervioso; manifestaciones neuromusculares; síndrome de guillain-barré

\section{Bibliografía}

1. Eduardo C. La pandemia por el nuevo coronavirus covid-19 [The novel coronavirus covid-19 pandemic]. Rev Fac Cien Med Univ Nac Cordoba. 2020 Mar 18;77(1):1-3. Spanish. doi: 10.31053/1853.0605.v77.n1.27935.

2. Zhao H, Shen D, Zhou H, Liu J, Chen S. Guillain-Barré syndrome associated with SARS-CoV-2 infection: causality or coincidence? Lancet Neurol. 2020 May;19(5):383-384. doi: 10.1016/S1474-4422(20)30109-5

3. Herman C, Mayer K, Sarwal A. Scoping review of prevalence of neurologic comorbidities in patients hospitalized for COVID-19. Neurology. 2020 Apr 28:10.1212/WNL.0000000000009673. doi: 10.1212/WNL.0000000000009673.

4. Guidon AC, Amato AA. COVID-19 and neuromuscular disorders. Neurology. 2020 Apr 13;10.1212/WNL.0000000000009566. doi: 10.1212/WNL.0000000000009566. 